\title{
Should non-movement specialists refer patients for SPECT-DaTSCAN?
}

\author{
Agata Gajos ${ }^{1}$, Janusz Dąbrowski², Małgorzata Bieńkiewicz ${ }^{2}$ Anna Płachcińska ${ }^{3}$, \\ Jacek Kuśmierek ${ }^{2}$, Andrzej Bogucki ${ }^{1}$ \\ ${ }^{1}$ Department of Extrapyramidal Diseases, Medical University of Łódz, Łódź, Poland \\ ${ }^{2}$ Department of Nuclear Medicine, Medical University of Łódź, Łódź, Poland \\ ${ }^{3}$ Department of Quality Control and Radiological Protection, Medical University of Łódź, Łódź, Poland
}

\begin{abstract}
Background. SPECT with radioligand DaTSCAN (SPECT-DaTSCAN) is a sensitive tool used for assessing the functional integrity of the presynaptic part of the nigrostriatal dopaminergic system. The procedure is useful whenever there is a need to distinguish between neurodegenerative parkinsonism and other parkinsonian syndromes in subjects with equivocal signs and symptoms. It can be assumed that the neurologist's decision to perform SPECT-DaTSCAN depends on his or her experience and skill in the diagnosis of parkinsonian and tremor syndromes.
\end{abstract}

Aims. To assess the accuracy of referrals to SPECT-DATSCAN made by non-movement disorders specialists.

Material and methods. Sixty seven patients referred for SPECT-DaTSCAN by a general neurologist were studied. In all subjects, a movement disorder specialist performed the neurological examination, collected medical history, and analysed previous treatments and the results of diagnostic tests.

Results. Evaluation carried out by a movement disorder specialist did not confirm an indication for SPECT-DaTSCAN in 31 patients (46.3\%). General neurologists needed support for clinical diagnosis with SPECT-DaTSCAN most frequently in subjects with parkinsonism even though they were presenting a full-blown disease manifestation and even though the patients met the diagnostic criteria for Parkinson's disease or one of the atypical parkinsonian syndromes.

Conclusions. Our presented results probably reflect the limited experience of general neurologists in the evaluation of parkinsonian syndromes and tremor. The use of SPECT-DaTSCAN by non-movement disorders specialists is associated with a significant risk of overuse of this tool. To minimise this risk, the skills of general neurologists in diagnosing parkinsonian and tremor syndromes should be improved. Moreover, patients should be provided with access to movement disorders specialists.

Key words: SPECT-DaTSCAN, referrals, non-movement disorders specialists, movement disorders specialists (Neurol Neurochir Pol 2019; 53 (2): 138-143)

\section{Introduction}

${ }^{123}[\mathrm{I}]$ ioflupane $\left(\left[{ }^{123} \mathrm{I}\right]\right.$-fluoropropyl CIT, ${ }^{123} \mathrm{I}$-FP-CIT, DaTSCAN ${ }^{\circ}$, GE Healthcare) is a dopamine transporter (DAT) radioligand for single-photon emission tomography (SPECT). Degeneration of dopaminergic neurons in the substantia nigra results in reduced DAT concentration within the striatum, and therefore SPECT-DaTSCAN is used as a marker of the integrity of the presynaptic part of the nigrostriatal dopaminergic system [1-7].

DaTSCAN was approved by EMA in Europe in 2000 and by FDA in the USA in 2011 for the differential diagnosis of parkinsonian tremor and essential tremor (ET) and of Alzheimer's disease $(\mathrm{AD})$ and diffuse Lewy body disease (LBD) [8-10]. In clinical practice, SPECT-DaTSCAN has wider applications in that it allows for differential diagnosis between

Address for correspondence: Agata Gajos, Department of Extrapyramidal Diseases, Medical University of Łódz, Łódź, Poland, e-mail: agata-gajos@wp.pl 
neurodegenerative parkinsonisms on the one hand, and parkinsonian syndromes without the presynaptic involvement of the nigrostriatal dopaminergic system on the other hand.

It can be assumed that the neurologist's decision to perform SPECT-DaTSCAN depends both on his or her experience and skill in the diagnosis of parkinsonian syndromes as well as on his or her knowledge of the usefulness and limitations of this technique. The aim of our paper was to assess the accuracy of referrals to SPECT-DATSCAN made by non-movement disorders specialists.

\section{Material and methods}

At the Department of Nuclear Medicine, we introduced a routine procedure according to which the indications for all patients referred for SPECT-DaTSCAN were verified by one of two neurologists ( $\mathrm{AG}$ or $\mathrm{AB}$ ) with more than 15 years of experience in Parkinson's disease and other movement disorders (> 50 patients seen per month). In all subjects, a neurological examination was performed, a medical history was carefully collected, and previous treatments and the available results of diagnostic tests were analysed.

Consecutive patients referred by a general neurologist from July 2016 to December 2017 were included in this present analysis.

The results presented in this paper are a retrospective analysis of data from routine practice and so there was no need to apply for the approval of the Bioethical Committee.

\section{Results}

Sixty seven patients referred for SPECT-DaTSCAN by a general neurologist were studied. Evaluation carried out by a movement disorder specialist did not confirm the indications for dopaminergic functional imaging in 31 patients (Group A), but found it to be reasonable in the other 36 subjects (Group B).

\section{Group A. Patients with unconfirmed}

indications for SPECT-DaTSCAN study

In this group of 31 patients (Tab. 1), the largest subgroup consisted of 22 patients (age 52-91 years, disease duration 1-16 years) who were referred for SPECT-DaTSCAN with an indication formulated by a general neurologist as „suspicion of PD”. In the opinion of a movement disorder specialist, the observed clinical symptoms were fully sufficient to diagnose PD in 12 subjects (aged 52-84, disease duration 1-5 years). All of them met the UK Brain Bank main diagnostic criteria for PD [12]: bradykinesia plus one or more of rigidity, tremor, or postural instability. Moreover, nine of these 12 (age 52-84 years, symptoms duration 2-5 years) presented with between one and four of the supportive prospective positive criteria [12]: unilateral onset, rest tremor, progressive disorder, persistent asymmetry affecting the side of greatest onset, excellent (70$100 \%$ ) response to levodopa, and 1-4 years duration of illness.

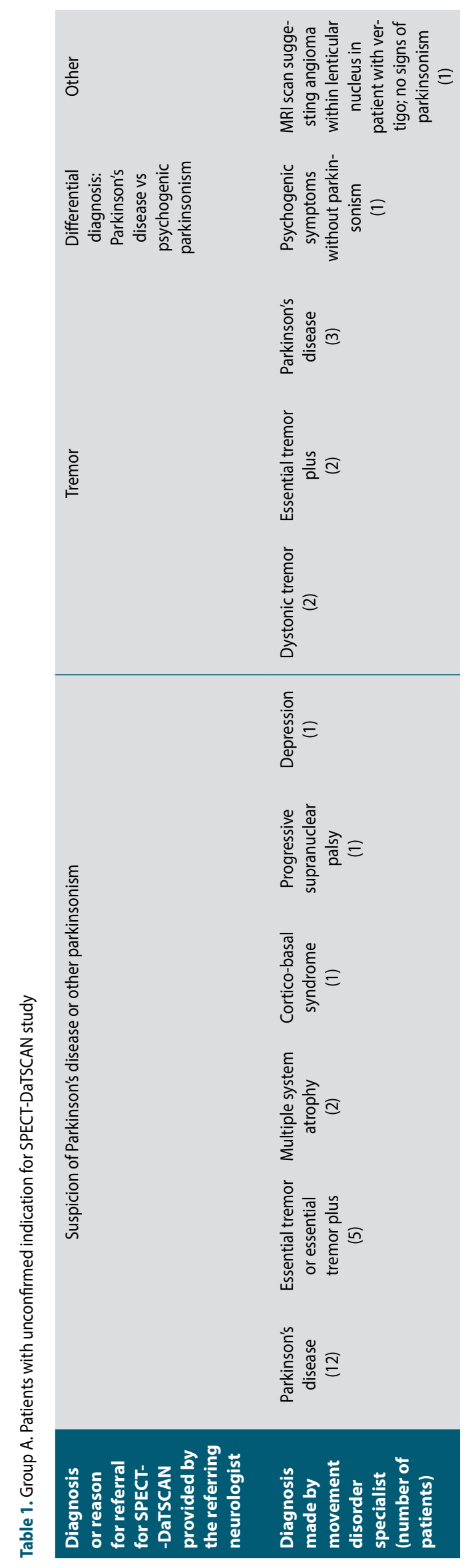


ET [13] was diagnosed in three patients (two female and one male, 58-70 years) with 3-6 years history of symmetrical bilateral upper limb action and postural tremor. All these patients had also developed head tremor, but no signs of parkinsonism were observed. In two male subjects without parkinsonian signs, a diagnosis of essential tremor plus [13] was made. The first of these, a 78 year-old patient with a 16 years history of tremor, presented also with impaired tandem gait. The second of them, a 91 year-old subject, had suffered from kinetic and postural upper limb symmetric tremor and head "no-no" tremor for six years. Impairment of tandem gait was found, and his mother had also suffered from tremor.

In Group A there were also four patients with unquestionable parkinsonian syndrome and other accompanying signs that allowed us to recognise one of the atypical parkinsonisms.

Two of these subjects met the diagnostic criteria for probable multiple system atrophy (MSA) [14, 15]. A 54 year-old female had a three-year history of progressive, more or less symmetrical, bradykinesia and rigidity which did not respond to levodopa. She presented also with dysarthria, dysphagia, laryngeal stridor, cortical myoclonus of the fingers (polyminimyoclonus), ataxic gait, postural instability, Babinski sign and orthostatic hypotension manifesting with dizziness. In the second patient, a 63 year-old man, bradykinesia, rigidity (right $\geq$ left) and postural, rest and action tremor (right $\geq$ left) had progressed for four years, and these symptoms did not respond to levodopa. There was a history of REM behaviour disorder, dysphagia, urinary incontinence, and orthostatic hypotension. He presented moreover with cerebellar dysarthria, laryngeal stridor, tendency for a drooping head, ataxia and Babinski sign.

The next patient in this subgroup, a 67 year-old woman with a four-year disease duration presented with asymmetrical (right $\geq$ left) rigidity and bradykinesia with dystonic posture and alien limb syndrome on the right, apraxia dysgraphoesthesia of the right upper limb, and mild cognitive impairment. She met the diagnostic criteria of probably corticobasal degeneration syndrome (CBS) [16].

A 68 year-old patient with a four-year history of falling backwards (observed from the first year of the disease), presented with - rather symmetrical - bradykinesia and rigidity, neck stiffness, tendency to retrocollis and vertical gaze palsy. Brain MRI scans showed midbrain atrophy. Probable progressive supranuclear palsy (PSP) - PSP with Richardson's syndrome - was diagnosed according to MDS diagnostic criteria [17].

Finally, there was a 78 year-old woman without any parkinsonian signs with diagnosis of depression made by a psychiatrist.

Among the patients with unconfirmed indications for the SPECT-DaTSCAN study there were seven subjects who were referred by a general neurologist due to tremor.

In two female subjects (age 71 and 62 years, disease duration 15 and 4 years, respectively) dystonic tremor was diagnosed by the movement disorder specialist. In neither of them were there parkinsonian signs, while the examination revealed the presence of mild torticollis and upper limbs dystonia.

The next two females (78 and 68 years, disease duration 7 and 6 years, respectively) presented with slightly asymmetric action tremor of upper limbs, impaired tandem gait and - in the first of them - memory impairment. No parkinsonian signs were present. Essential tremor plus [13] was diagnosed in both these cases.

Two female patients and one male patient (age 51, 55 and 57 years, disease duration 3,1 and 4 years, respectively) showed a full-blown asymmetric parkinsonian syndrome (including asymmetric rest tremor) which met the core UK Brain Bank criteria for Parkinson's disease. In a male subject with a fouryear history of rest tremor, a good response to levodopa had been well documented.

A 39 year-old female (disease duration 1 year) was referred for SPECT-DaTSCAN as part of a differential diagnosis of psychogenic parkinsonism and $\mathrm{PD}$. The patient reported many subjective complaints including pain and numbness located bilaterally within the feet, ankles and knees. Peripheral neuropathy and pathology within the spinal cord had been previously excluded. She did not meet the diagnostic criteria for restless legs syndrome. Her neurological examination was normal and, most of all, there were no parkinsonian signs. In the movement disorder specialist's opinion, the patient's symptoms were of psychogenic origin.

The last patient in Group A, a 61 year-old female, reported vertigo with onset one year ago. Her neurological examination was normal, and brain MRI scans suggested angioma within lenticular nucleus. According to the neurologist referring this subject for SPECT-DaTSCAN, the purpose of the study was „the assessment of dopaminergic system”.

In patients with unconfirmed indications for SPECTDaTSCAN, the study was not performed. The exception was two patients with a diagnosis of atypical parkinsonism made by the movement disorders specialist; in these cases, the SPECT-DaTSCAN result was abnormal.

\section{Group B. Patients with confirmed indications for SPECT-DaTSCAN}

In the opinion of movement disorder specialists, the decision to perform the SPECT-DaTSCAN study was well-founded in 17 female and 18 male subjects (Tab. 2).

In 16 patients (nine women and seven men, age range 37-57 years; disease duration 1-4 years), the imaging study was a part of the process of differential diagnosis between essential tremor and PD. At this point, in all these cases, it was indeed difficult or impossible to make a diagnosis solely on the basis of clinical symptoms.

The next subgroup consisted of four male patients (age range 61-77, disease duration 2-6 years), who required differential diagnosis between vascular and neurodegenerative parkinsonism, especially PD. 

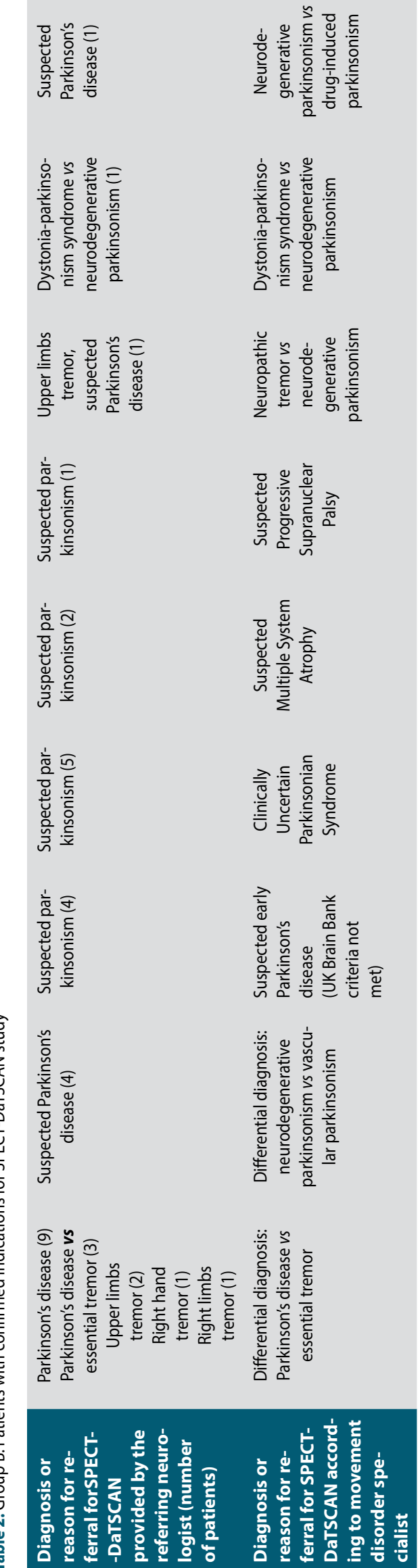

Three men and one woman (aged 37-51 years, disease duration 1-2 years) suspected by the referring neurologist for Parkinson's disease, presented with different combinations of two core UK Brain Bank criteria parkinsonian signs, but they did not meet the diagnosis of PD.

Furthermore, five patients (three females and two males, age 58-67, disease duration 3-7 years) were classified as Clinically Uncertain Parkinsonian Syndrome (CUPS). They did not meet the diagnostic criteria for PD or any atypical parkinsonism, and there was a poor response to levodopa, so drug-induced parkinsonism was excluded.

Two patients (a 41 year-old female and a 37 year-old male, both with three years of symptoms duration) with uncertain symptoms suggesting MSA and a man (62 years, disease duration 2 years) presenting with scant symptoms and suspected of PSP were also qualified for SPECT-DaTSCAN to support the pre-scan diagnosis.

The need to perform the SPECT study was confirmed also in a 69 year-old woman (symptoms duration 2 years) with axonal polyneuropathy and asymmetric $(\mathrm{L}>\mathrm{P})$ postural, rest and action tremor. The aim was to confirm or to exclude the co-occurrence of neurodegenerative parkinsonism.

In another 39 year-old female patient, writer's cramp had developed when she was about 20 and subsequently dystonia spread to the contralateral upper limb. Neurological examination revealed unilateral bradykinesia and rigidity. Neither dystonia nor parkinsonian signs responded to levodopa. Differential diagnostics between dystonia-parkinsonism syndrome and neurodegenerative parkinsonism required imaging of the nigrostriatal dopaminergic system.

Finally, we accepted a 59 year-old woman who had been treated with neuroleptics for many years before acute, asymmetric parkinsonism had developed. The purpose of the SPECT study in this case was to exclude the co-existence of nigrostriatal degeneration.

\section{Discussion}

The concentration and distribution of DAT within the striatum reflect the number of active dopaminergic neurons in the substantia nigra. SPECT-DaTSCAN is a sensitive tool for assessing the functional integrity of the presynaptic part of the nigrostriatal dopaminergic system [1-7]. DaTSCAN is currently approved in Europe and the USA for use in the differential diagnosis between PD tremor and essential tremor as well as for differentiation between AD and LBD. However, in clinical practice, SPECT-DaTSCAN is used much more widely and may be useful whenever there is a need to distinguish between neurodegenerative parkinsonism (PD, MSA, PSP, CBS) and parkinsonian syndromes not resulting from damage to the dopaminergic system in subjects with equivocal signs and symptoms (DIP, PP, VP) [18].

SPECT-DaTSCAN is a very sensitive functional marker of the lesion of the presynaptic part of the nigrostriatal system 
and - according to new MDS diagnostic criteria [19] - normal dopaminergic imaging is an absolute exclusion criterion for PD. SPECT-DaTSCAN is not recommended as a part of routine diagnostic procedure because in patients who meet the UK Brain Bank diagnostic criteria for PD the results of dopaminergic imaging are abnormal [12]. The same applies to atypical parkinsonisms if the main signs of parkinsonian syndrome are present [14-17]. Moreover, SPECT-DaTSCAN is completely useless in the differential diagnosis of neurodegenerative parkinsonisms: PD, MSA, PSP and CBS [20-23].

The usefulness of SPECT-DaTSCAN in the practice of movement disorders centres has been confirmed by numerous reports. At referral movement disorders centres, the diagnosis and treatment were changed in more than half of the patients as a result of dopaminergic imaging [24-25].

SPECT-DaTSCAN is a relatively expensive procedure, and therefore the rational use of this diagnostic tool is of particular importance. The British NICE 2017 [11] guidelines underline that this procedure may eliminate the costs of unnecessary treatment of patients with a false positive diagnosis based on a neurological examination. On the other hand, it has been suggested that SPECT-DaTSCAN should not be used in all subjects suspected of $\mathrm{PD}$, and it is recommended that this procedure "should be available to specialists with expertise in its use and interpretation".

Until 2018, the availability of SPECT-DaTSCAN in Poland was very limited. However, at the Department of Nuclear Medicine in Medical University of Łodź a number of patients could be tested without any payment from their side (the procedure was funded by the Regional Branch of the National Health Fund). Patients could be referred by both movement disorder specialists and neurologists with no special interest in movement disorder (i.e. non-movement disorders specialists, general neurologists).

There are no reference centres for neurological subspecialties within the Polish healthcare system. Patients with Parkinson's disease and other movement disorders are diagnosed and treated by general neurologists who have differing levels of experience in this area and who are usually not able to refer patients to a movement disorders specialist for consultation. There is therefore a risk that the SPECT-DaTSCAN will be overused in this situation.

To the best of our knowledge, this is the first study to have assessed the correctness of non-movement disorders specialists' decision to use SPECT-DaTSCAN in routine clinical practice.

We evaluated 67 patients referred by general neurologists for dopaminergic imaging to the Department of Nuclear Medicine. In 31 (46.3\%) of these subjects, in the opinion of movement disorders specialists, it was possible to make a diagnosis without a SPECT study, solely on the basis of neurological examination and data from medical history. Non-movement disorders specialists needed support for clinical diagnosis with SPECT-DaTSCAN most often in cases of parkinsonism. It should be noted that this concerned patients who had been ill for several years and had full-blown disease symptoms. The same problem occurred in subjects with tremor. This, in our opinion, most likely reflects the limited experience of this group of neurologists in the diagnosis of tremor and parkinsonian syndromes.

The general neurologists who referred patients to the Department of Nuclear Medicine were not obliged to provide alternative diagnoses which, according to them, needed differentiation using SPECT-DaTSCAN. Therefore, we could not assess to what extent the choice of SPECT-DaTSCAN as a tool to resolve the neurologist's uncertainty regarding the pre-scan diagnosis considered this technique's basic application, i.e. the distinction between degenerative and non-degenerative parkinsonisms.

In the group of patients with confirmed indications for the SPECT-DaTSCAN study, there were several subjects in whom the signs and symptoms revealed by neurological examination were insufficient to make a diagnosis. In these cases, the abnormal result of imaging could confirm the presence of nigrostriatal degeneration and enable early diagnosis. This was a finely balanced decision, because in these cases longer clinical observation would probably also allow for diagnosis.

The presented results show that the use of SPECTDaTSCAN by non-movement disorders specialists is associated with a significant risk of overuse of this diagnostic tool. To minimise this risk, general neurologists' skills in diagnosing parkinsonian syndromes should be improved, and at the same time patients should be provided with access to a movement disorders specialist. In addition, broad access to DaTSCAN financed by the National Health Fund should be connected to an educational programme addressed to all neurologists.

Acknowledgements: The study was funded by the Medical University of Lodz (grant 503/7-127-01/503-51-002).

Conflict of interest: None declared

\section{References}

1. Abi-Dargham A, Gandelman MS, DeErausquin GA, et al. SPECT imaging of dopamine transporters in human brain with iodine-123-fluoroalkyl analogs of beta-CIT. J Nucl Med. 1996; 37(7): 1129-1133, indexed in Pubmed: 8965183.

2. Seibyl JP, Marek K, Sheff K, et al. Test/retest reproducibility of iodine123-betaCIT SPECT brain measurement of dopamine transporters in Parkinson's patients. J Nucl Med. 1997; 38(9): 1453-1459, indexed in Pubmed: 9293807.

3. Booij J, Habraken JB, Bergmans P, et al. Imaging of dopamine transporters with iodine-123-FP-CIT SPECT in healthy controls and patients with Parkinson's disease. J Nucl Med. 1998; 39(11): 1879-1884, indexed in Pubmed: 9829575.

4. Huang WS, Chiang YH, Lin JC, et al. Crossover study of (99m)TC-TRODAT-1 SPECT and (18)F-FDOPA PET in Parkinson's disease patients. J Nucl Med. 2003; 44(7): 999-1005, indexed in Pubmed: 12843212. 
5. Kish SJ, Shannak K, Hornykiewicz O. Uneven pattern of dopamine loss in the striatum of patients with idiopathic Parkinson's disease. Pathophysiologic and clinical implications. N Engl J Med. 1988; 318(14): 876-880, doi: 10.1056/NEJM198804073181402, indexed in Pubmed: 3352672.

6. Seibyl J, Russell D, Jennings $D$, et al. The role of neuroimaging in the early diagnosis and evaluation of Parkinson's disease. Minerva Med. 2005; 96(5): 353-364, indexed in Pubmed: 16227950.

7. Brooks DJ, Pavese N, Brooks DJ, et al. Recent imaging advances in the diagnosis and management of Parkinson's disease. F1000 Med Rep. 2009; 1(4): 614-628, doi: 10.3410/M1-82, indexed in Pubmed: 20948696.

8. Website EMApifD. (2000).

9. Darcourt J, Booij J, Tatsch K, et al. EANM procedure guidelines for brain neurotransmission SPECT using (123)l-labelled dopamine transporter ligands, version 2. Eur J Nucl Med Mol Imaging. 2010; 37(2): 443-450, doi: 10.1007/s00259-009-1267-x, indexed in Pubmed: 19838702.

10. DaTscan Fpif. 2011.

11. National Institute for Health and Clinical Excellence. Parkinson's Disease in Adults: Diagnosis and Management. London: National Institute for Health and Care Excellence (UK); 2017 Jul.

12. Hughes AJ, Daniel SE, Kilford L, et al. Accuracy of clinical diagnosis of idiopathic Parkinson's disease: a clinico-pathological study of 100 cases. J Neurol Neurosurg Psychiatry. 1992; 55(3): 181-184, indexed in Pubmed: 1564476.

13. Bhatia KP, Bain P, Bajaj $\mathrm{N}$, et al. Tremor Task Force of the International Parkinson and Movement Disorder Society. Consensus Statement on the classification of tremors. from the task force on tremor of the International Parkinson and Movement Disorder Society. Mov Disord. 2018; 33(1): 75-87, doi: 10.1002/mds.27121, indexed in Pubmed: 29193359.

14. Gilman S, Wenning GK, Low PA, et al. Second consensus statement on the diagnosis of multiple system atrophy. Neurology. 2008; 71(9): 670-676, doi: 10.1212/01.wnl.0000324625.00404.15, indexed in Pubmed: 18725592.

15. Stankovic I, Krismer F, Jesic A, et al. Movement Disorders Society MSA (MODIMSA) Study Group. Cognitive impairment in multiple system atrophy: a position statement by the Neuropsychology Task Force of the MDS Multiple System Atrophy (MODIMSA) study group. Mov
Disord. 2014; 29(7): 857-867, doi: 10.1002/mds.25880, indexed in Pubmed: 24753321.

16. Armstrong MJ, Litvan I, Lang AE, et al. Criteria for the diagnosis of corticobasal degeneration. Neurology. 2013; 80(5): 496-503, doi: 10.1212/WNL.0b013e31827fOfd1, indexed in Pubmed: 23359374.

17. Höglinger GU, Respondek G, Stamelou M, et al. Movement Disorder Society-endorsed PSP Study Group. Clinical diagnosis of progressive supranuclear palsy: The movement disorder society criteria. Mov Disord. 2017; 32(6): 853-864, doi: 10.1002/mds.26987, indexed in Pubmed: 28467028.

18. Isaacson SH, Fisher S, Gupta F, et al. Clinical utility of DaTscan ${ }^{\mathrm{TM}}$ imaging in the evaluation of patients with parkinsonism: a US perspective. Expert Rev Neurother. 2017; 17(3): 219-225, doi: 10.1080/147371 75.2017.1256205, indexed in Pubmed: 27813429.

19. Postuma RB, Berg D, Stern M, et al. MDS clinical diagnostic criteria for Parkinson's disease. Mov Disord. 2015; 30(12): 1591-1601, doi: $10.1002 / m d s .26424 .$.

20. Vlaar AMM, de Nijs T, Kessels AGH, et al. Diagnostic value of 123lioflupane and 123l-iodobenzamide SPECT scans in 248 patients with parkinsonian syndromes. Eur Neurol. 2008; 59(5): 258-266, doi: 10.1159/000115640, indexed in Pubmed: 18264015.

21. Kim YJ, Ichise M, Ballinger JR, et al. Combination of dopamine transporter and D2 receptor SPECT in the diagnostic evaluation of PD, MSA, and PSP. Mov Disord. 2002; 17(2): 303-312, indexed in Pubmed: 11921116.

22. Perju-Dumbrava LD, Kovacs GG, Pirker S, et al. Dopamine transporter imaging in autopsy-confirmed Parkinson's disease and multiple system atrophy. Mov Disord. 2012; 27(1): 65-71, doi: 10.1002/ mds.24000, indexed in Pubmed: 22102521.

23. Filippi L, Manni C, Pierantozzi M, et al. 123I-FP-CIT in progressive supranuclear palsy and in Parkinson's disease: a SPECT semiquantitative study. Nucl Med Commun. 2006; 27(4): 381-386, doi: 10.1097/01. mnm.0000202858.45522.df, indexed in Pubmed: 16531926.

24. Oravivattanakul S, Benchaya L, Wu G, et al. Dopamine Transporter (DaT) Scan Utilization in a Movement Disorder Center. Mov Disord Clin Pract. 2016; 3(1): 31-35, doi: 10.1002/mdc3.12261, indexed in Pubmed: 30363515.

25. Sadasivan S, Friedman JH. Experience with DaTscan at a tertiary referral center. Parkinsonism Relat Disord. 2015; 21(1): 42-45, doi: 10.1016/j.parkreldis.2014.10.022, indexed in Pubmed: 25465746. 